\title{
Relevance and paucity of evidence: a dental perspective on personal protective equipment during the COVID-19 pandemic
}

\author{
Jennifer E. Gallagher, ${ }^{* 1}$ Ilona Johnson, ${ }^{2}$ Jos H. Verbeek, ${ }^{3}$ Janet E. Clarkson ${ }^{4,5}$ and Nicola Innes ${ }^{6}$
}

\section{Key points}

Outlines contemporary evidence on personal protective equipment for health professionals.
Explores its relevance for dental professionals, with practical recommendations for action.
Outlines the critical lack of relevant evidence and where further research is required involving dental personnel, procedures and in dental settings.

\begin{abstract}
The global COVID-19 pandemic, caused by the SARS-CoV-2 virus, has highlighted the importance of personal protective equipment (PPE) for health and social care personnel. This is a really important issue for dentistry, where we place great emphasis on infection control and universal precautions, given the nature of care provided.

Cochrane have recently updated their review of PPE for preventing highly infectious diseases due to exposure to contaminated body fluids in healthcare staff. It examined evidence on which type of full body PPE and which method of donning (putting on) or doffing (removing) are most effective, while having the least risk of contamination or infection for healthcare workers, as well as which training methods increase compliance with PPE protocols. The objective of this paper is to raise awareness of the above review of PPE, its findings and their relevance to dentistry as outlined in the Cochrane Oral Health website.

The available evidence comes from healthcare generally, mostly involving simulation exercises, and is of low or very low certainty. None of the evidence specifically comes from dentistry.

The findings in relation to the nature of PPE, methods of donning and doffing, and the importance of training are all of practical relevance to dentistry. Research is critically important to provide evidence for future decision making in support of patients and staff.
\end{abstract}

\section{Introduction}

\section{COVID-19 pandemic}

The global COVID-19 pandemic, caused by the SARS-CoV-2 virus, ${ }^{1}$ has highlighted the

'Dean for International Affairs, Newland-Pedley Professor of Oral Health Strategy, Honorary Consultant in Dental Public Health, Faculty of Dentistry, Oral \& Craniofacial Sciences, King's College London, Bessemer Road, London, SE5 9RS, UK; ${ }^{2}$ Reader and Honorary Consultant in Dental Public Health, School of Dentistry, Applied Clinical

Research and Public Health, College of Biomedical and Life Sciences, Cardiff University, Heath Park, Cardiff, CF14 4XY, UK; ${ }^{3}$ Cochrane Work Review Group, Academic Medical Centre, University of Amsterdam, Amsterdam, Netherlands; "Professor of Clinical Effectiveness \& Joint Coordinating Editor Cochrane Oral Health, University of Dundee \& University of Manchester, UK; ${ }^{5}$ Child Dental and Oral Health, School of Dentistry, University of Dundee Park Place, Dundee, DD1 4HR, UK; ${ }^{6}$ Professor of Paediatric Dentistry, Child Dental and Oral Health, School of Dentistr University of Dundee, Park Place, Dundee, DD1 4HR, UK.

${ }^{*}$ Correspondence to: Jennifer Gallagher

Email address: jenny.gallagher@kcl.ac.uk

Refereed Paper.

Accepted 1 June 2020

https://doi.org/10.1038/s41415-020-1843-9 importance of personal protective equipment (PPE) for health and social care personnel.

It is important to acknowledge from the outset that PPE has proved a contentious issue across health and social care generally across the United Kingdom (UK), and beyond, and is one that will need to be resolved practically moving forwards. This is of critical importance to dentistry, where we have historically placed great emphasis on infection control and universal precautions, given the nature of care provided.

\section{Transmission of SARS-CoV-2 virus}

Based on evidence to date, the World Health Organisation suggests that transmission of the SARS-CoV-2 virus is mainly via respiratory droplet and contact routes, with transmission being possible through aerosol generating procedures (AGPs). ${ }^{2}$ Droplet transmission occurs when a person is in close contact (within $1 \mathrm{~m}$ ) with someone who has respiratory symptoms (for example, coughing or sneezing) and is therefore at risk of having his/her mucosae (mouth and nose) or conjunctiva (eyes) exposed to potentially infective respiratory droplets. Person-toperson transmission routes for COVID-19 disease can involve direct and indirect contact, ${ }^{2}$ and it is important to recognise that this coronavirus is present in saliva. ${ }^{3,4,5}$

SARS-CoV-2 is a novel coronavirus and, because of the nature of their occupation, healthcare workers (HCWs) are often at greater risk of infection than the general population. ${ }^{6}$ Deaths of colleagues across healthcare have highlighted these risks, leading to concern and calls for greater protection for staff. Appropriate PPE offers an important way of reducing the risk of infection during the provision of healthcare.

\section{Cochrane review of PPE}

An important Cochrane review published in April 2020, ${ }^{7}$ and recently updated, ${ }^{8}$ examines 'personal protective equipment, for preventing 
highly infectious diseases due to exposure to contaminated body fluids, in healthcare staff'? It reviewed contemporary evidence on 'which type of full-body PPE and which method of donning (putting on) or doffing (removing) PPE have the least risk of contamination or infection for HCW, and which training methods increase compliance with PPE protocols. ${ }^{8}$ The evidence from this review is of great importance where there is a risk of highly infectious diseases, and even though COVID-19 is no longer considered to be a high consequence disease in the $\mathrm{UK},{ }^{9}$ its findings remain relevant to the current pandemic ${ }^{10}$ and continue to be updated.

The objective of this paper is to raise awareness of the findings of the above review of $\mathrm{PPE}^{8}$ and explore their relevance to dentistry, building on our commentary presented on the Cochrane Oral Health website. ${ }^{10}$

\section{Cochrane and COVID-19}

Cochrane synthesises the best available evidence using rigorous methodology to answer specific research questions, thus drawing on the body of evidence available to inform decision-making, ${ }^{11}$ using thorough methods. ${ }^{12}$ The COVID Cochrane group are prioritising questions related to COVID-19, ${ }^{13}$ reviewing the literature and synthesising wide-ranging data in a matter of weeks rather than the usual extended period of at least two years. ${ }^{14}$ Groups have prioritised this task and are collaborating where interventions are common across profession groups or health conditions. It involves rapid peer review of protocols and search strategies, working many extra hours to complete them as quickly as possible without compromising their quality, with final peer review and editing before publication. One example is the fast-tracking of this review of PPE for all HCWs. ${ }^{7,8}$ Current work by Cochrane Oral Health includes rapid reviews of mouthwashes and nasal sprays, and methods to reduce aerosols produced during AGPs, as well as a rapid review of international dental guidelines for return to dental services. ${ }^{15}$

\section{Relevance of Cochrane PPE review to dentistry}

The PPE review questions for HCWs are relevant to the practice of dentistry and all dental professionals working in clinical settings, including dentists, dental hygienists, dental nurses, dental therapists, orthodontic therapists, dental technicians and clinical dental technicians, along with reception and cleaning staff and practice managers. ${ }^{10}$ Clinical members of the dental team work in close proximity, usually face-to-face, with patients and often for sustained periods of time. Over and above the risk associated with proximity to potentially infected individuals, during routine care, they are exposed to saliva and blood and carry out AGPs (for example, use of high-speed air rotors and ultrasonic scalers). For COVID-19, personal protection entails preventing droplets from entering their mouth, nose or eyes and preventing them from contaminating the skin elsewhere. This makes the findings of the review highly relevant to the dental profession. Although there is no evidence to say that dental procedures increase the likelihood of patients coughing, if they do, clinicians are in close proximity. This further increases the chance of aerosol and droplet generation, as well as infected material settling on environmental surfaces and on PPE.

\section{Available evidence: relevance to dentistry}

While the search included a broad range of HCWs, only 24 studies (controlled studies, either randomised or non-randomised) were included, most from simulation exercises and none directly associated with dentistry. ${ }^{8}$ In the midst of an acute situation where the primary evidence is difficult, and probably impossible to generate with enough speed to be useful, a judgement has to be made on how confident we are that the findings of this review can be applied to the dental care setting. However, in the absence of direct evidence from studies situated in a dental setting, we have to take note of, and realistically apply, the general evidence.

It is important to note that 'the certainty of the evidence presented in the review, ${ }^{8}$ across all comparisons, was judged to be low or very ${ }^{10 w^{10}}$ for a range of reasons. This related to the paucity of research addressing each of the questions, together with the fact that much of the available research involved simulations of exposure rather than research in real-life conditions, small sample sizes, high or unclear risk of bias and insufficient detail on whether the PPE used fitted international standards for protective clothing. ${ }^{8,10}$ Furthermore, most of the research understandably used harmless microbes or fluorescent markers rather than microbes or viruses of concern. ${ }^{8,10}$ Nonetheless, its key findings, against which the questions were relevant to dentistry, are important given the above caveats (Box 1).

While it is important to acknowledge that 'members of the dental team are very experienced in the use of standard PPE, most work within primary care settings, and may be less familiar with the more extensive forms of $\mathrm{PPE},{ }^{10}$ although this is rapidly changing.

The Cochrane review suggests that 'covering more of the body' leads to 'better protection.' This included gowns providing better protection than just an apron. ${ }^{8}$ The evidence suggests that added coverage provided by a coverall (one-piece suit) when compared with a gown comes at a cost in terms of increased 'difficulty in doffing' such PPE. ${ }^{8}$ While there were initial concerns that challenges with doffing increased the risk of contamination, current evidence suggests that, in 'more recently introduced full-body PPE ensembles, there may be no difference in contamination.8 ${ }^{8}$ The review also suggests that 'PPE made from more breathable material may help increase user satisfaction, with little impact on contamination.' ${ }^{10}$

The head and neck areas of the dental team are particularly at risk for HCWs during clinical dental procedures. ${ }^{10}$ Thus, ensuring PPE coverage is adequate to protect these areas is an important aspect of its effectiveness; in addition, 'better fitting PPE' in this region, 'sealed gown and glove combinations' to protect wrists, and certain design features such as 'tabs to "grab" during doffing and donning' may help to reduce the risk of contamination. ${ }^{8,10}$ Overall, PPE should provide full coverage but not be too cumbersome.

It is important not to make the mistake of assuming that just 'having' face masks and other elements of enhanced PPE is 'good enough'. Dental professionals also need to be aware of the risks of contamination associated with donning and, in particular, doffing PPE. Space for these processes will need to be considered as part of dental surgery organisation where care is being delivered, as well as the time involved. Training in donning and doffing is particularly important for dental teams who may not wear this type of PPE for routine practice or who may need to learn new, safer habits carefully. The presence of an observer, in particular for doffing PPE, should be considered. Teams should consider face-toface training opportunities as they may reduce the likelihood of errors, alongside computer 


\section{Box 1 Key findings: systematic review of PPE for $\mathrm{HCWs}^{8}$}

Review question: 'To evaluate which type of full-body PPE and which method of donning or doffing PPE have the least risk of contamination or infection for HCWs, and which training methods increase compliance with PPE protocols.'

Key findings:

- Coverage: there is better protection from covering more parts of the body, but this has to be balanced against the possible increase in risk of contamination associated with difficulty donning or doffing as well as less user comfort

- Materials: PPE made of more breathable material may lead to similar contamination as more waterproof materials, but have greater user satisfaction

- Design: Risk of contamination may be decreased by using PPE with design modifications such as sealed glove/gown combinations, well-fitting gown around the neck, and tabs on gloves and masks to aid removal

- Process of donning and doffing: following Centres for Disease Control and Prevention (CDC) guidance, particularly during doffing, one-step glove and gown removal and double-gloving may lead to less contamination

- Sanitation: glove disinfection before doffing with quaternary ammonium or bleach (but not alcoholbased hand rub) may decreased contamination

- Training: training in donning and doffing PPE face-to-face may reduce errors in follownig procedures more successfully than folder- or video-based training.

- Compliance: spoken instructions during donning and particularly doffing PPE may reduce contamination and increase compliance

Research: further research is recommended during this outbreak of COVID-19, with long-term follow-up. Simulations, randomised controlled trials and real-life evidence on agreed outcomes are required.

simulation or videos which may also support these skills. ${ }^{8,10}$

\section{Research required}

The Cochrane review makes a strong case for building evidence to inform decisions on the 'most appropriate manageable protection', including 'modifications for HCWs'. ${ }^{8} \mathrm{We}$ concur that this is essential for dentistry, if dental teams are to deliver care safely. ${ }^{10}$ It also 'provides helpful insights on the research required, and the importance of registering and coordinating research with comparable outcomes. ${ }^{10}$ We need to consider how we can best do this across the four nations of the UK and connect with our global partners who are also facing the same issues.

\section{Dental care centres}

There is an opportunity, during this COVID19 outbreak, to use the natural experimental setting that dental care centres provide to create the evidence we need on health outcomes and personnel involved. ${ }^{10}$ These opportunities include, but are not limited to, the issues of viral transmission rates, those related directly to the training, education and use of PPE, as well as how this affects patient care. ${ }^{8,10}$

\section{Trials in dental care settings}

Safe provision of dental care requires a deep understanding of pathogen transmission and how it relates to the various types of care provided; for example, AGPs and non-AGPs. ${ }^{10}$ Trials using high-quality and standardised methodology considering the spread and settle of demonstration pathogens or surrogate measures in dental settings are key, and these should consider the array of different procedures that are considered to generate aerosols. It is worth noting that fluorescent dyes or harmless bacteria and viruses have been used for much of the higher quality simulated research in the past. ${ }^{8}$ They should include all relevant settings and consider single and multiple surgeries as well as laboratories and domiciliary care. ${ }^{10}$

Studies should be well-designed and of sufficient sample size, with agreed outcomes. ${ }^{8}$ Crossover studies should be conducted where possible. Details of education and training, fit testing, equipment used (including standards), dental examination and special investigations and procedure(s), length of appointment, nature of patient(s), technique of dental care, donning and doffing processes, environment and a range of outcomes should be recorded. ${ }^{10}$

\section{Standard versus enhanced PPE}

We need to know the most appropriate type of PPE for clinical encounters with different potential exposure levels. For example, it would be helpful to confirm whether standard PPE is adequate for an oral examination while more elaborate PPE, even with its drawbacks, is necessary for treatments where aerosols are actively generated during the procedure. Also, are the types of PPE required for dental professionals carrying out routine care during peak phases of the COVID-19 pandemic still required for the post-peak phase? In addition, there needs to be an understanding of the amount of time that recommended PPE can be worn comfortably. Simulation exercises involving comparison of different levels of PPE will be particularly helpful to inform standard requirements for different aspects of dental care. ${ }^{10}$ This includes the effect of masks, face shields and goggles. ${ }^{10}$ Furthermore, we will also need to consider shielded patients and the best way of affording them the necessary protection as well as staff.

While we have considered the review in a dental context, we currently lack critical knowledge on whether viral load and shedding are similar in asymptomatic individuals ${ }^{16}$ and to what extent this presents a risk in dentistry. ${ }^{17}$ Evidence in these areas would allow better understanding of appropriate PPE. We also need to deepen our understanding of aerosol generation generally, ${ }^{18}$ as well as specifically in relation to viruses in dental settings.

\section{Future considerations}

Given the burden of oral disease ${ }^{19}$ and the evidence that transmission of SARS-CoV-2 by seemingly well individuals (pre-symptomatic and asymptomatic), we need to seriously consider how our patient and population needs are best met for the future. Models indicate that pre-symptomatic individuals alone may account for $30-62 \%$ of events (confirmed COVID cases)..$^{20,21,22,23}$

It is important to remember that most patients attending for dental care will be COVID-negative. Important questions are being debated regarding the nature and extent of universal precautions, and whether we should adopt a precautionary principle to protect our dental teams and patients until more is known and these can be confidently relaxed. Measures such as self-isolation and testing are now being suggested to reduce risk associated with planned and urgent care in 
hospital settings. ${ }^{24}$ PPE should be considered after risk assessment and as just one issue in a larger preventive approach, including aerosol, droplet and splatter reduction and ventilation. Risk reduction must be considered along with other major challenges to our staff's health and wellbeing, including the nature of care and the complex business of dentistry. We do not have all the answers about universal precautions for the future, but all dental professionals will need to take action in identifying and managing risk in line with national guidance and learning from our colleagues around the world. We have to be able to justify our actions in managing risk, and collect evidence and be prepared to adapt where necessary.

\section{In conclusion}

Having PPE is important, but so is wearing it properly and removing it safely; it is important to remember that PPE is just one way of protecting dental professionals and patients, all of which require careful consideration and research to inform our journey back to what may become a 'new normal'.

\section{Acknowledgements}

Professor A. M. Glenny, Cochrane Oral Health.

\section{References}

1. World Health Organisation. Naming the coronavirus disease (COVID-19) and the virus that causes it. 2020. Available at https://www.who.int/emergencies/ diseases/novel-coronavirus-2019/technical-guidance/ naming-the-coronavirus-disease-(covid-2019)-and-thevirus-that-causes-it (accessed June 2020).

2. World Health Organisation. Modes of transmission of virus causing COVID-19: implications for IPC precaution recommendations. 2020. Available at https://www. who.int/news-room/commentaries/detail/modes-oftransmission-of-virus-causing-covid-19-implicationsfor-ipc-precaution-recommendations (accessed June 2020).

3. To K KW, Tsang O TY, Leung WS et al. Temporal profiles of viral load in posterior oropharyngeal saliva samples and serum antibody responses during infection by SARSCOV2: an observational cohort study. Lancet Infect Dis 2020; 20: 565-574.

4. To K K-W, Tsang O T-Y, Yip C C-Y et al. Consistent detection of 2019 novel coronavirus in saliva. Clin Infect Dis 2020; DOI: 10.1093/cid/ciaa149.

5. Khurshid Z, Asiri F Y I, AI Wadaani H. Human Saliva: Non-Invasive Fluid for Detecting Novel Coronavirus (2019-nCoV). Int J Environ Res Public Health 2020; 17: 2225 .

6. Chou R, Dana T, Buckley D I, Selph S, Fu R, Totten A M. Epidemiology of and Risk Factors for Coronavirus Infection in Health Care Workers. Ann Intern Med 2020; DOI: 10.7326/M20-1632

7. Verbeek J H, Rajamaki B, ljaz S et al. Personal protective equipment for preventing highly infectious diseases due to exposure to contaminated body fluids in healthcare staff. Cochrane Database Syst Rev 2020; DOI: 10.1002/14651858.CD011621.pub4.

8. Verbeek J H, Rajamaki B, ljaz S et al. Personal protective equipment for preventing highly infectious diseases due to exposure to contaminated body fluids in healthcare staff. Cochrane Database Syst Rev 2020; DOI: 10.1002/14651858.CD011621.pub5.

9. UK Government. High consequence infectious diseases (HCID) - Status of COVID-19. 2020. Available at https:// www.gov.uk/guidance/high-consequence-infectiousdiseases-hcid (accessed June 2020).

10. Johnson I, Gallagher J E, Verbeek J H, Innes N P T. Personal protective equipment: a commentary for the dental and oral health care team. 2020. Available at https://oralhealth.cochrane.org/news/personalprotective-equipment-commentary-dental-and-oralhealth-care-team (accessed June 2020).

11. Cochrane Library. About Cochrane Reviews. 2020. Available at https://www.cochranelibrary.com/about/ about-cochrane-reviews (accessed June 2020).
12. Cochrane Training. Cochrane Handbook for Systematic Reviews of Interventions. 2020. Available online at https://training.cochrane.org/handbook (accessed June 2020).

13. Cochrane Methods. Rapid Reviews. 2020. Available online at https://methods.cochrane.org/rapidreviews/ welcome (accessed June 2020).

14. Andersen M Z, Gülen S, Fonnes S, Andresen K, Rosenberg J. Half of Cochrane reviews were published more than two years after the protocol. I Clin Epidemiol 2020; 124: 85-93.

15. Cochrane Oral Health. 2020. Available at https:// oralhealth.cochrane.org/ (accessed June 2020).

16. Zou L, Ruan F, Huang M et al. SARSCoV2 Viral Load in Upper Respiratory Specimens of Infected Patients. N Engl J Med 2020; 382: 1177-1179.

17. Rautemaa R, Nordberg A, Wuolijoki-Saaristo K, Meurman J H. Bacterial aerosols in dental practice - a potential hospital infection problem? J Hosp Infect 2006; 64: 76-81.

18. Greenhalgh T. Editor's commentary: Rapid reviews of PPE - an update. 2020. Available at https://www.cebm. net/covid-19/editors-commentary-rapid-reviews-ofppe-an-update/ (accessed June 2020).

19. Marcenes W, Kassebaum N J, Bernabé E et al. Global Burden of Oral Conditions in 1990-2010: A Systematic Analysis. J Dent Res 2013; 92: 592-597.

20. Ferretti L, Wymant C, Kendall M et al. Quantifying SARSCOV2 transmission suggests epidemic control with digital contact tracing. Science 2020; DOI: 10.1126/ science.abb6936

21. Li R, Pei S, Chen B et al. Substantial undocumented infection facilitates the rapid dissemination of novel coronavirus (SARS-CoV2). Science 2020; 368: 489-493.

22. Ganyani T, Kremer C, Chen D et al. Estimating the generation interval for COVID-19 based on symptom onset data. 2020. Preprint. Available online at https://www.medrxiv.org/ content/10.1101/2020.03.05.20031815v1 (accessed June 2020).

23. He X, Lau E H Y, Wu P et al. Temporal dynamics in viral shedding and transmissibility of COVID-19. Nat Med 2020; 26: 672-675.

24. NHS England. Operating framework for urgent and planned services in hospital settings during COVID-19. 2020. Available at https://www.england.nhs.uk/ coronavirus/wp-content/uploads/sites/52/2020/05/ Operating-framework-for-urgent-and-plannedservices-within-hospitals.pdf (accessed June 2020). 